\title{
Photoacoustics of soil organic matter: comparison of various IR modalities for soil fractions of various agrogenesis
}

Krivoshein $\mathrm{P}^{1}{ }^{1}$, Volkov D. ${ }^{1,2}$, Mikheev I. ${ }^{1}$, Rogova O. ${ }^{2}$, Proskurnin M. ${ }^{1}$

${ }^{1}$ Chemistry Department of M.V. Lomonosov Moscow State University, Moscow, Russia, 119991

${ }^{2}$ Department of Chemistry and Physical Chemistry of Soils, Dokuchaev Soil Science Institute, Moscow 119017, Russia

doi: 10.36291/HIT.2019.krivoshein

The studies of anthropogenesis influence on physical and physicochemical properties of soils experience a notable shift. Apart from traditional approaches to assessing the bulk properties, the new information level is to determine soil fractions and aggregate structures including the changes at meso- and microaggregate levels. IR photoacoustic (PA) spectroscopy is an instrument for delicate measurement and modeling of the dependence of soil properties on its physical and elemental composition, moisture, porosity, and density. The PA effect is based on the absorption of IR radiation by the sample followed by the heat transfer to the environment. Pressure waves in surrounding gas are detected by a sensitive microphone providing high sensitivity of measurements [1]. FTIR PA spectrometry has several features like simpler sample preparation, small amounts of the test sample, and the ability to monitor the penetration of radiation in the sample.

In this study, the dependence of mid-IR PA spectra of chernozem soils of various agrogenesis conditions was studied and compared with ATR-FTIR spectroscopy. PA modality provides measurements of soil aggregates up to 2-5 $\mathrm{mm}$ without sample decomposition, which is a unique property of this modality. The spectra quality does not change for various size fractions. PA spectra clearly show the organic matter region from 1800 to $1300 \mathrm{~cm}^{-1}$ with some differences in the composition. Below $1300 \mathrm{~cm}^{-1}$, the bands are weak in comparison, which is different from PA measurements of other types of samples like engineered nanomaterials. In our opinion, it requires theoretical modeling and experimentally studying model systems. For soil, PA and ATR modalities are complementary - while ATR provides high sensitivity for the mineral part of soil, the organic part is rather poorly revealed, and vice versa for PA measurements. ATR spectra of soil fractions in the mineral part are different, and FTIR PA and ATR measurements may be used together for studying soil degradation and recultivation processes.

Acknowledgements. The authors acknowledge the financial support of The Russian Science Foundation, grant no. 19-13-00117. 